\title{
Metastasis from Penile Squamous Cell \\ Carcinoma to Brain: A Case Report and Review of Literature
}

\author{
Hanish Bansal' Ashwani Chaudhary ${ }^{1}$ Dipesh Batra ${ }^{1}$ Rohit Jindal ${ }^{2}$ \\ ${ }^{1}$ Department of Neurosurgery, Dayanand Medical College and \\ Hospital, Ludhiana, Punjab, India \\ ${ }^{2}$ Department of General Surgery, Dayanand Medical College and \\ Address for correspondence Dr. Hanish Bansal, MCh, 10-b Udham \\ Singh Nagar, Ludhiana, Punjab, India \\ (e-mail: y2khanish@rediffmail.com).
} Hospital, Ludhiana, Punjab, India

Indian J Neurosurg 2018;7:164-167
Abstract
Keywords
- metastasis
- penile squamous cell carcinoma
- brain

We report a very rare case of a 63-year-old man with penile carcinoma who developed brain metastasis. Brain metastasis from penile carcinoma is very rare, and only six cases have been reported so far as per our literature review. In view of controlled primary tumor and absence of systemic disease, tumor was excised and the patient remained clinically controlled till 3 months after follow-up.

\section{Introduction}

Metastasis from penile squamous cell carcinoma to the brain is extremely rare. We conducted a thorough literature review and found out that only six cases have been reported so far out of which three were found on autopsy. We report a case of a 63-year-old man with penile squamous cell carcinoma who developed brain metastasis. Survival benefit of surgical resection of brain metastasis is limited to patients with controlled primary tumor, absence of systemic disease, and good performance status. Patients with a single-brain metastasis and positive prognostic features benefit more from surgical resection followed by whole-brain radiation therapy compared with whole-brain radiation therapy alone.

\section{Case Report}

A 63-year-old man presented with history of 15 days of significantly discomforting headaches associated with multiple episodes of vomiting. Eight months ago, he was diagnosed with penile squamous cell carcinoma and underwent a partial penectomy with inguinal lymph node dissection at another hospital. The patient had already received external beam radiation therapy and four cycles of chemotherapy. The penile stump was clean. He was asymptomatic till his current illness. On examination, he was drowsy and had papilledema on fundus examination. Contrast-enhanced computed tomography (CT) of the brain revealed well-defined heterogeneously enhancing mass lesion in the right cerebellar hemisphere (3.6 into $2.5 \mathrm{~cm}$ ) with transtentorial and tonsillar herniation ( $\mathbf{- F i g . 1}$ ). CT of the head also revealed marked obstructive hydrocephalus due to compression of the fourth ventricle ( - Fig. 2). Magnetic resonance imaging (MRI) of the brain revealed similar findings. Differential of a secondary tumor versus a malignant primary tumor was considered. Ventriculoperitoneal shunt was performed in view of obstructive hydrocephalus. Postoperatively the patient became fully conscious. Complete metastatic workup was done including positron emission tomography that did not reveal lesion anywhere except the brain. In view of controlled primary tumor and on suspicion of cerebellar mass being metastatic, complete excision of mass was done via suboccipital craniectomy after 5 days. Histology was consistent with metastatic carcinomatous deposit ( - Fig. 3). He subsequently received whole-brain radiotherapy. At the 3-month follow-up, he remained clinically controlled.

\section{Discussion}

Most common intracranial tumor in adults is brain metastasis, occurring in 8 to $10 \%$ of cancer patients, and it causes

(C2018 Neurological Surgeons' Society of India
License terms

(요 (1) $\odot \circledast$
July 12,2016

accepted

January 23, 2017

published online

May 19, 2017
DOI https://doi.org/

10.1055/s-0037-1601359.

ISSN 2277-954X. 


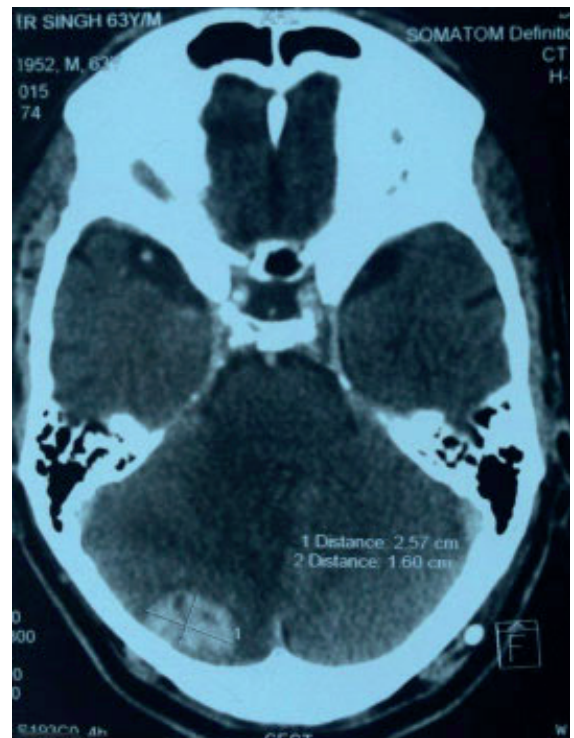

Fig. 1 Contrast CT of the head showing right cerebellar enhancing space-occupying lesion.

significant cancer-related morbidity and mortality worldwide. These are usually found at the junction of the gray and white matters, with circumscribed margins. In a presence of a known primary cancer, detection of intracranial mass raises the suspicion of a metastasis. An intracranial mass is often life threatening and warrants prompt treatment. Lung cancer followed by breast cancer, melanoma, renal cancer, and colon cancer account for most of the cases of brain metastases. However, any malignant tumor can metastasize to the brain. ${ }^{1}$

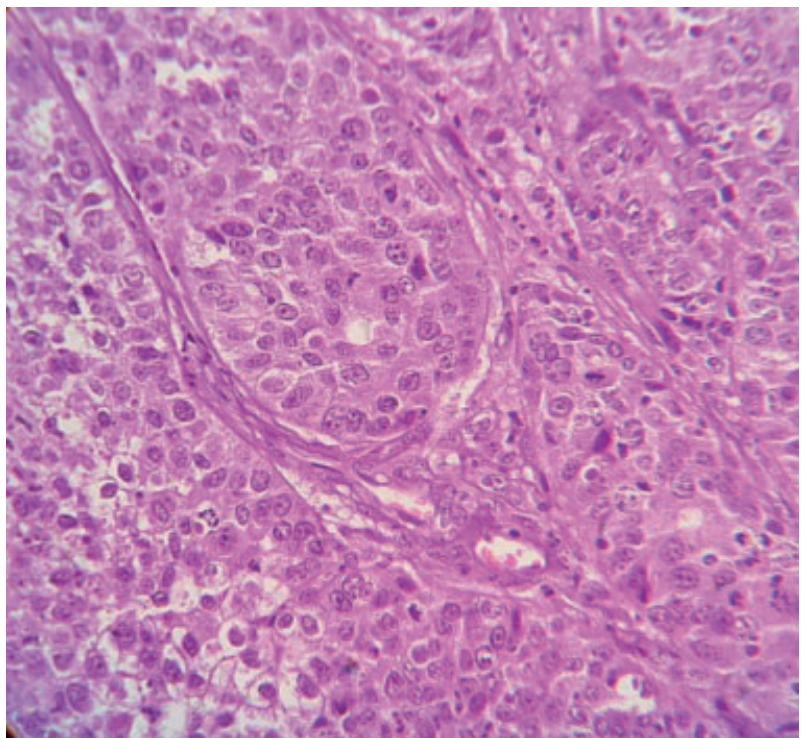

Fig. $3 \mathrm{H} \&$ E section showing sheets and nests of squamous epithelial cells infiltrating brain parenchyma.

Patients with symptomatic brain metastasis usually have involvement of the lung (lung being the first capillary bed passed by circulating tumor cells after crossing right side of the heart). Tumors such as renal cell carcinoma, colon, and breast carcinomas generally produce single metastases whereas malignant melanoma and lung carcinoma generally produce multiple secondary lesions. ${ }^{2}$

Metastasis from penile carcinoma to central nervous system is extremely rare, and only six cases with brain metastases have been described to date ( - Table 1$)$. Majority

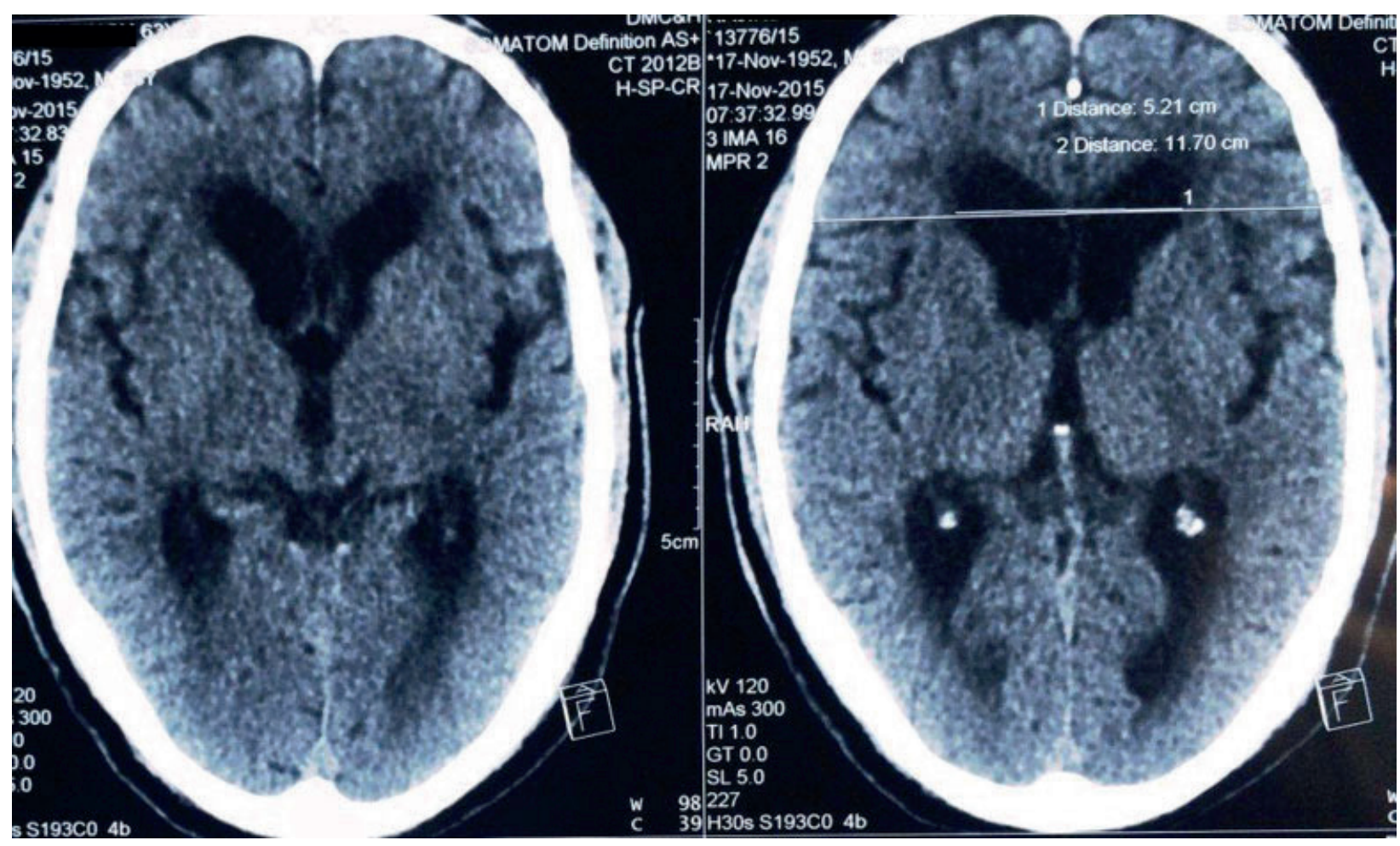

Fig. 2 CT of the head showing marked obstructive hydrocephalus. 
Table 1 Reported cases of brain metastasis from penile squamous cell carcinoma

\begin{tabular}{|l|l|l|l|}
\hline S. No. & Author & Case profile & \\
\hline 1. & ${\text { Chaux et } \mathrm{al}^{6}(2011)}^{\text {Autopsy study of } 14 \text { patients with }}$ & $\begin{array}{l}2 \text { patients had brain metastasis on } \\
\text { autopsy }\end{array}$ \\
\hline 2. & Swierz et $\mathrm{al}^{7}(1992)$ & 20-year-man with penile carcinoma & Brain metastasis on autopsy \\
\hline 3. & ${\text { Moiyadi et } \mathrm{al}^{8}(2010)}^{\text {Middle-aged man with penile carcinoma }}$ & Left perisylvian metastasis \\
\hline 4. & Skripuletz et $\mathrm{al}^{9}(2010)$ & $\begin{array}{l}\text { 68-year-old man with poorly differentiated } \\
\text { penile squamous cell carcinoma }\end{array}$ & $\begin{array}{l}\text { Brain metastasis with leptomeningeal } \\
\text { and subarachnoid space involvement }\end{array}$ \\
\hline 5. & ${\text { Lutterbach et } \mathrm{al}^{10}(2005)}^{\text {35-year-old man with early-stage penile }}$ & Right frontal lobe metastasis \\
\hline
\end{tabular}

of penile malignant tumors are squamous cell carcinomas. The progression of disease is locoregional, and about onethird of patients with penile cancer die from disseminated metastatic disease.

Penile carcinoma typically affects middle-aged to older men (those between 50 and 70 years of age) and in decreasing order of frequency, and it affects glans (48\%), prepuce (21\%), glans and prepuce (9\%), coronal sulcus (6\%), and shaft $(<2 \%)^{3}$. The world's highest prevalence rates are found in developing countries such as India and Brazil. ${ }^{4}$ Factors such as exposure to the human papilloma virus, lack of neonatal circumcision, and exposure to tobacco have been implicated as risk factors for penile carcinoma. ${ }^{5}$

Chaux et al in an autopsy study of 14 patients with penile squamous cell carcinoma (13 cases from Memorial Sloan-Kettering Cancer Center, New York, and 1 from the National Cancer Institute, Asuncion, Paraguay) found brain metastasis in two patients at autopsy and concluded that the regional (inguinal and pelvic) and systemic lymph nodes (retroperitoneal) are the most frequent sites of metastasis. Metastatic deposits in their study were seen in the lymph nodes (nine cases); liver (seven cases); lungs (six cases); heart (five cases); adrenals, bone, and skin (three cases each); thyroid (two cases); and pancreas, spleen, and pleura (one case each). ${ }^{6}$

Swierz et al reported a rare case of penile carcinoma in a 20-year-old man in whom metastasis was found in the heart and brain on autopsy.

Moiyadi et al in 2010 reported a case of middle-aged man with penile carcinoma who had undergone a partial penectomy with right groin node dissection. Two years later he developed headache and right upper limb monoparesis and was found to have well-circumscribed, lobulated intra-axial mass in the left perisylvian area on MRI. Histology revealed metastasis from squamous cell carcinoma. ${ }^{8}$

Skripuletz reported a case of a 68-year-old man who presented with poorly differentiated penile squamous cell carcinoma who underwent subtotal penectomy and adjuvant chemotherapy and radiation. Eleven months later the patient developed brain metastases and infiltration of the leptomeninges and subarachnoid space by carcinoma cells. ${ }^{9}$

Most patients develop brain metastasis late during the course of metastatic cancer. Lutterbach et al reported a case of a 35-year-old man with early-stage penile squamous cell carcinoma who underwent circumcision and bilateral inguinal lymphadenectomy. Subsequently the patient developed metastases at multiple sites, including in the kidney, adrenal gland, retroperitoneal lymph nodes, lung, and brain (right frontal lobe) for which he underwent multiple resections, whole-brain radiotherapy, and several chemotherapy regimens. $^{10}$

Contrast-enhanced MRI is investigation of choice to define total number of metastatic nodules and to look for evidence of leptomeningeal disease. Without treatment, prognosis of patients with intracranial metastasis is very poor and the median survival is only 4 to 7 weeks. Systemic steroids alone may prolong survival to approximately 2 months and improve neurologic function by reducing peritumoral edema. Patients with a single-brain metastasis and positive prognostic features benefit more from surgical resection followed by whole-brain radiation therapy compared with whole-brain radiation therapy alone.

Survival benefit of surgical resection of brain metastasis is limited to patients with controlled primary tumor, absence of systemic disease, and good performance status. There is limited role of chemotherapy in the treatment of brain metastases. The goals of surgery in the management of brain metastases include immediate relief of neurologic symptoms due to increased intracranial pressure, histologic confirmation of diagnosis when the diagnosis is in doubt (like in penile cancers in which intracranial metastases are uncommon), local control of disease, reduction in focal neurologic deficits and seizures, and a rapid steroid taper.

Radiosurgery allows the treatment of brain metastases in almost any location, and survival following radiosurgery is comparable to that achieved with surgery. Stereotactic radiosurgery combined with whole-brain radiation therapy is superior to whole-brain radiation therapy alone in terms of survival.

Source(s) of Support

None.

\section{Presentation at Meeting}

None.

\section{Conflict of Interest}

None. 


\section{References}

1 Gramada FN, Indrei A, Iencean SM, Poeata I, Dumitrescu G, Miron L. Management of unknown origin cerebral metastases. Romanian Neurosurg 2011;18:60-67

2 Biswas G, Bhagwat R, Khurana R, Menon H, Prasad N, Parikh PM. Brain metastasis-evidence based management. J Cancer Res Ther 2006;2(01):5-13

3 Pow-Sang MR, Benavente V, Pow-Sang JE, , et al . Cancer of the penis. Cancer Contr 2002;9(04):305-314

4 Gomez V, Cuesta L, Bretones F, et al. Inguinal lymphadenectomy assessment associated with penile carcinoma undergone new strategies for nodal staging. Case Reports Clin Med 2014;3:410-415

5 Caso JR, Rodriguez AR, Correa J, Spiess PE. Update in the management of penile cancer. Int Braz J Urol 2009;35 (04):406-415
6 Chaux A, Reuter V, Lezcano C, Velazquez E, Codas R, Cubilla AL. Autopsy findings in 14 patients with penile squamous cell carcinoma. Int J Surg Pathol 2011;19(02):164-169

7 Swierz J, Poznański J, Stawarz B. [Metastasis of penile cancer to the heart in a 20-year-old patient] [in Polish] Wiad Lek 1992;45(07-08): 314-316

8 Moiyadi AV, Tongaonkar HB, Bakshi GK. Symptomatic intracranial metastasis in penile carcinoma. Indian J Urol 2010;26(04):585-586

9 Skripuletz T, Pul R, Herrmann J, et al. Meningeal carcinomatosis from penile squamous cell carcinoma. J Neurooncol 2010;98(03):417-419

10 Lutterbach J, Pagenstecher A, Weyerbrock A, SchultzeSeemann W, Waller CF. Early-stage penile carcinoma metastasizing to brain: case report and literature review. Urology 2005;66 (02):432 